\title{
Test Bench Demonstration of the Geometric Wavefront Sensor
}

\author{
Sierra Hickman, Stephen Weddell, and Richard Clare \\ University of Canterbury, Christchurch, New Zealand
}

\begin{abstract}
This paper investigates the performance and optimization of the Geometric Wavefront Sensor (GWFS) in openloop wavefront sensing, along with the Curvature Wavefront Sensor (CWFS) and Shack-Hartmann Wavefront Sensor (SH-WFS). The GWFS uses a ray tracing process to calculate the displacement of intensity fluctuations from two defocused point source images. While similar to the CWFS, initial simulations and testing have shown the GWFS to have superior performance compared to the CWFS. Various parameters within the GWFS - such as the signal-to-noise ratio (SNR) sensitivity, the number of Radon angles, the virtual propagation distance, and the number of reconstruction modes - are explored on a laboratory test bench. We found that the GWFS wavefront estimate error experiences an inverse relationship to the SNR, a minimum of 5 Radon angles is required to accurately estimate the single Zernike mode wavefronts $\left(Z_{4}-Z_{15}\right)$, the virtual propagation distance is confined by ray crossing and Fresnel diffraction effects, and the number of reconstruction Zernike modes is limited by noise amplification and over-fitting. This paper demonstrates the capabilities of the GWFS, illustrates the resulting wavefront estimates, and confirms the superior performance of the GWFS compared to the CWFS. The optimized GWFS will be utilized at Mt. John University Observatory (MJUO) in New Zealand for satellite and space debris imaging and tracking.
\end{abstract}

Keywords: Wavefront sensing, Geometric wavefront sensor, Curvature wavefront sensor, adaptive optics, ShackHartmann

\section{INTRODUCTION}

Images of stars or other astronomical objects are distorted as their light travels through turbulence within Earth's atmosphere. ${ }^{1}$ Specifically, the atmospheric turbulence causes non-uniform phase shifts to the wavefront of light. ${ }^{2}$ Therefore the resolution of ground-based telescopes are limited not only by the optics of the telescope, but also by the turbulence overhead. ${ }^{3}$

Adaptive Optics (AO) ${ }^{4}$ and Deconvolution from Wavefront Sensing (DWFS) ${ }^{5}$ are techniques used on groundbased telescopes to overcome the blurring effects of the atmosphere and aim to achieve diffraction-limited imaging resolution. In both AO and DWFS, a Wavefront Sensor (WFS) estimates the phase aberrations introduced by the atmospheric turbulence. The accuracy of the WFS estimate limits the performance of the AO or DWFS system, therefore making the WFS a critical component for both imaging techniques.

A wavefront of light is composed of a complex field where the phase does not interact directly with matter. ${ }^{4}$ As a result, the WFS cannot directly measure the wavefront phase. Instead, the wavefront of interest is perturbed by a known aberration, which then the WFS records the response of the wavefront's intensity to deduce the wavefront phase. ${ }^{6}$ Depending upon the WFS, the response is related to either the slope or curvature of the wavefront phase. The overall shape of the wavefront phase is then estimated and reconstructed using a series of basis functions, typically Zernike polynomials. ${ }^{7}$

Two WFSs commonly used for AO and DWFS are the Shack-Hartmann WFS ${ }^{4,6}$ (SH-WFS) and the Curvature WFS $^{8}$ (CWFS). The SH-WFS subdivides the aperture plane of the telescope using a lenslet array to measure the local slope of the wavefront. The CWFS uses the intensity difference between two defocused images of the telescope aperture to measure the curvature of the wavefront.

Further author information: (Send correspondence to Sierra Hickman)

E-mail: sierra.hickman@pg.canterbury.ac.nz 
A comparatively new WFS, the Geometric WFS (GWFS), uses assumptions from geometric optics (ray tracing) to calculate the displacement of intensity fluctuations from two defocussed images of the telescope aperture. The GWFS is similar to the CWFS, as they both use the same defocussed images. However unlike the CWFS, the GWFS measures the slope of the wavefront instead of the curvature.

The GWFS was initially conceptualized by Van Dam in $2002,{ }^{9}$ and first demonstrated by Chew in $2003 .{ }^{10}$ However, it has only recently received significant interest. ${ }^{11-14}$ Pal et al. ${ }^{11}$ first used the GWFS on-sky in open-loop DWFS in 2016, while Colodro-Conde et al. ${ }^{14}$ were the first to use the GWFS (which they call Two Pupil Plane Positions Wavefront Sensor or TP3-WFS) in closed-loop AO in 2017. While similar to the CWFS, initial investigations have shown the GWFS to have superior performance over the CWFS. ${ }^{10,11,13}$ It is of interest to further validate these results, as well as to compare the GWFS's performance against the SHWFS in a controlled laboratory environment. It is also of importance to characterize and optimize the GWFS's performance capabilities for DWFS and AO due to the relatively new interest in the GWFS.

The GWFS is also of interest to the authors due to its potential for multi-conjugate and multi-object AO. Like the CWFS, the GWFS can potentially estimate wavefronts from multiple guide stars concurrently, allowing for atmospheric tomography. Weddell et al. have proposed, and are currently developing, an instrument for Mt. John University Observatory (MJUO) in Tekapo, New Zealand to utilize atmospheric tomography for space situational awareness. ${ }^{15}$ Specifically, the instrument will use AO and DWFS techniques to image and track satellites and space debris. The GWFS and CWFS are both candidates for this instrument due to their potential capabilities in atmospheric tomography and satellite imaging.

This paper investigates possibilities to optimize the performance of the GWFS in the laboratory, as well as to characterize the wavefront estimates of the GWFS against the SH-WFS and CWFS. In Section 2, we describe the mathematical background of the atmospheric imaging problem, detail the three relevant WFSs (GWFS, CWFS, and SH-WFS), and mention the Zernike polynomials and reconstruction algorithm used to create the wavefront estimates. Our optical setup, the data collection process, and the GWFS properties investigated are explained in Section 3. The wavefront estimate results are discussed in Section 4. Finally, in Section 5 we conclude and propose future research.

\section{BACKGROUND}

\subsection{Astronomical Imaging}

The blurred image of an astronomical object, $d(u, v)$, is mathematically described as the original unperturbed object, $o(u, v)$, convolved with the Point Spread Function (PSF) of the atmosphere, $h(u, v)$, or

$$
d(u, v)=o(u, v) \odot h(u, v)+n(u, v)
$$

where $n(u, v)$ is additive detector noise on the data. ${ }^{16}$ The $\odot$ operator denotes convolution, and $(u, v)$ are the telescope's focal plane coordinates. Deconvolution attempts to recover the original object by inverting Equation (1) using the observed blurred image, an estimate of the PSF, and assumptions of the detector noise.

The aperture plane of the telescope is described by the Fourier space of Equation (1),

$$
\mathcal{D}(x, y)=\mathcal{O}(x, y) \mathcal{H}(x, y)+\mathcal{N}(x, y)
$$

where $(x, y)$ are the normalized coordinates in the telescope's aperture plane. $\mathcal{H}(x, y)$ is equal to the autocorrelation of the generalized pupil function (also known as the complex field of the telescope aperture),

$$
\mathcal{H}(x, y)=S(x, y) \exp [j \phi(x, y)] \otimes S(x, y) \exp [j \phi(x, y)],
$$

where $j=\sqrt{-1}, S(x, y)$ is the telescope aperture shape, $\phi(x, y)$ is the wavefront phase across the aperture, and $\otimes$ is the correlation operator. ${ }^{2}$ Using the auto-correlation theorem and Fourier transform properties, ${ }^{17}$ the PSF is directly related to the wavefront phase by,

$$
h(u, v)=|\mathcal{F}[S(x, y) \exp [j \phi(x, y)]]|^{2} .
$$




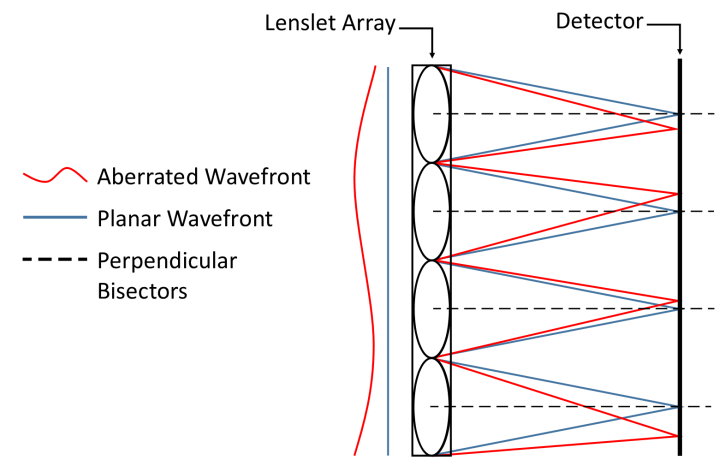

Figure 1: The SH-WFS with a planar wavefront and an aberrated wavefront. The dashed lines notate the perpendicular bisectors of the lenslets. ${ }^{20}$

A WFS produces an estimate of the wavefront phase, $\hat{\phi}(x, y)$. The estimated phase can either be applied to an optical corrector, such as a Deformable Mirror (DM) in AO, ${ }^{4}$ or used in DWFS by employing Equation (4) with a deconvolution algorithm, such as the Wiener filter, ${ }^{17}$ Lucy-Richardson, ${ }^{18}$ or blind. ${ }^{19}$ While neither AO nor DWFS can completely remove all of the PSF from the original object, they minimize the effects caused by the turbulence in astronomical imaging.

\subsection{Shack-Hartmann Wavefront Sensor}

A diagram of the SH-WFS subdividing a wavefront is depicted in Figure 1. A low resolution image of the astronomical object is formed at the focal plane of each lenslet. Without atmospheric turbulence aberrating the planar wavefront from an astronomical object, each lenslet would form a focused spot along the perpendicular bisector of the lenslet, as seen by the blue wavefront in Figure 1. However if the atmospheric turbulence aberrates the wavefront and introduces an average wavefront slope across an individual lenslet, the low resolution image is displaced from the perpendicular bisector, as seen by the red wavefront Figure 1.

Local wavefront slopes are calculated by measuring the displacement of the centroid of the low resolution image from the lenslet's perpendicular bisector in the $\mathrm{x}$ and $\mathrm{y}$ directions. If the detector has $2 P \mathrm{x} 2 Q$ pixels per lenslet-image and a pixel width of $\Delta$, the centroid displacement estimate is,

$$
\begin{aligned}
& m_{x}=\left[\sum_{p=-P+1}^{P} \sum_{q=-Q+1}^{Q} I(p \Delta, q \Delta)\left(p \Delta-\delta_{u}\right)\right] /\left[\sum_{p=-P+1}^{P} \sum_{q=-Q+1}^{Q} I(p \Delta, q \Delta)\right] \\
& m_{y}=\left[\sum_{p=-P+1}^{P} \sum_{q=-Q+1}^{Q} I(p \Delta, q \Delta)\left(p \Delta-\delta_{v}\right)\right] /\left[\sum_{p=-P+1}^{P} \sum_{q=-Q+1}^{Q} I(p \Delta, q \Delta)\right],
\end{aligned}
$$

where $m_{x}$ is the displacement in the $x$ direction, $m_{y}$ is the displacement in the $y$ direction, and $\left(\delta_{u}, \delta_{v}\right)$ is the pixel position of the perpendicular bisector. ${ }^{21}$ The wavefront across the entire telescope aperture is then reconstructed by either stitching the local wavefront slope measurements from each lenslet or using reconstruction algorithms, such as least-squared (discussed in Section 2.6).

\subsection{Curvature Wavefront Sensor}

Created in 1988 by Roddier, the CWFS was designed specifically for astronomy. ${ }^{8}$ The CWFS measures the curvature of the wavefront across the telescope aperture and the radial slope at the edge of the aperture. Two defocused aperture images, $I_{1}(x, y)$ and $I_{2}(x, y)$, are captured at a distance $\pm l$ from the telescope focal plane, as 


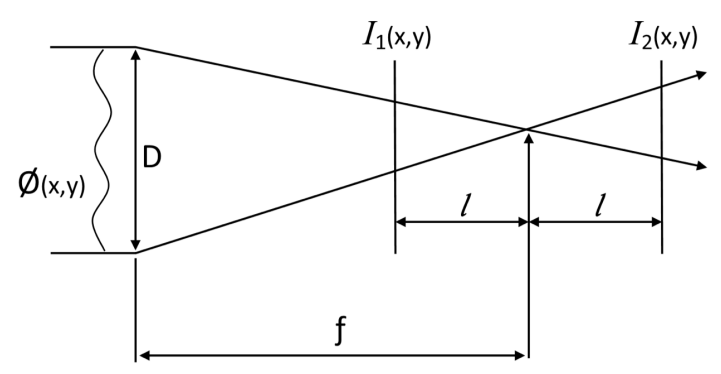

Figure 2: The configuration of the CWFS and GWFSs. The intra-focal, $I_{1}(x, y)$, and extra-focal $I_{2}(x, y)$, defocused images are taken at $\pm l$, respectively.

depicted in Figure 2. An aberration in the wavefront phase will appear as opposing intensity changes in the two images. The difference in intensity distributions is then used to estimate the wavefront phase aberration using,

$$
\frac{I_{1}(x, y)-I_{2}(-(x, y))}{I_{1}(x, y)+I_{2}(-(x, y))}=\frac{f(f-l)}{2 l}\left[\frac{\partial \phi}{\partial n}\left(\frac{f(x, y)}{l}\right) \delta_{c}-P \nabla^{2} \phi\left(\frac{f(x, y)}{l}\right)\right],
$$

where $f$ is the focal length, $P$ is the transmission function of the telescope aperture, $\delta_{c}$ is the linear impulse distribution around the aperture edge, $\partial \phi / \partial n$ is the radial slope, and $\nabla^{2}$ is the Laplacian operator. ${ }^{22}$ From Equation (7), it is worth noting that $I_{2}(x, y)$ must be inverted in order to have the same orientation as $I_{1}(x, y)$. The defocused images are also normalized to separate the wavefront from scintillation effects.

\subsection{Geometric Wavefront Sensor}

The GWFS setup is identical to the CWFS seen in Figure 2; two defocused images are taken at $\pm l$ from the focal plane. However the CWFS uses the difference of the two intensity profiles to determine the second derivative of the wavefront across the telescope aperture, while the GWFS measures the displacement of the intensity fluctuations to calculate the first derivative.

Light from an aberrated wavefront will converge and diverge in a non-uniform manner, depending upon the shape of the wavefront. This non-planar wavefront yields intensity fluctuations, similar to light "dancing" on the bottom of a swimming pool. Through conservation of light, the total wavefront intensity must be constant between the two defocused images,

$$
C_{I_{1}}=\int_{-\infty}^{\infty} I_{1}(x) d x=\int_{-\infty}^{\infty} I_{2}(x) d x=C_{I_{2}}
$$

where $I_{1}(x)$ and $I_{2}(x)$ are the intensity distributions of the two defocused images along the $x$ axis. ${ }^{10}$ An area of light divergence in one image will have the same total intensity as the corresponding convergent area in the other image, however the two areas will differ in size, as seen in Figure 3(a). Assuming geometric optics, paths of individual light rays can be traced by measuring the displacement of the corresponding intensity regions in the defocused images. Extending the path of the ray between the planes of the two defocused images to the telescope aperture determines the shape of the wavefront. At large propagation distances, $l$, a problem arises due to the light rays from different areas of the telescope aperture crossing before reaching the detectors. When the rays cross, it becomes impossible to unambiguously extend the light path back to the aperture and recover the wavefront slope. ${ }^{10}$

For a one dimensional wavefront, light ray $i$ at position $x_{A}$ in the intra-focal image traveling to position $x_{B}$ in the extra-focal image has a wavefront slope along the $x$ axis, $W_{x}$, described by ${ }^{9}$

$$
W_{x}\left(\frac{x_{A}(i)+x_{B}(i)}{2}\right)=\frac{x_{B}(i)-x_{A}(i)}{2 l} .
$$

The slope is found for every light ray across the telescope aperture. The slopes are then stitched together to reconstruct the estimated 1D wavefront. Radon transforms ${ }^{23}$ convert $2 \mathrm{D}$ wavefronts into $1 \mathrm{D}$ profile slices at multiple angles, similar to a CT scan or MRI. An example of a Radon transform on a 2D signal is seen in Figure $3(\mathrm{~b})$. 


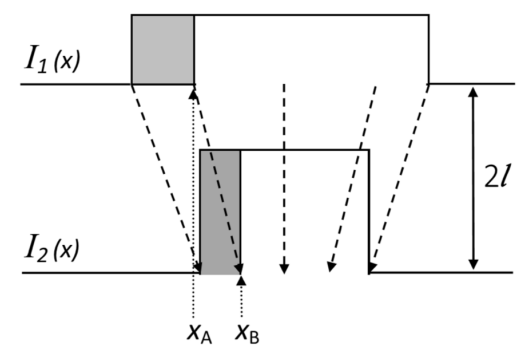

(a) Geometric propagation of light. The shaded region in both imaging planes has the same total light intensity. ${ }^{10}$

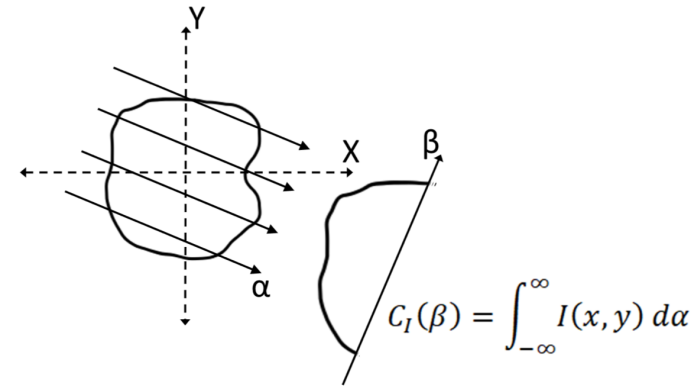

(b) A Radon transform using one angle, $\alpha$, on a single 2D signal. ${ }^{10}$

Figure 3: Visualization of the theory and the signal processing of the GWFS.

\subsection{Zernike Polynomials}

As mentioned at the end of Section 2.1, a WFS estimated phase, $\hat{\phi}(x, y)$, is used to reduce the effects from the atmospheric turbulence in both AO and DWFS. While it is possible to reconstruct the phase of the wavefront at every point across the telescope aperture, it is mathematically convenient to represent the phase as a sum of infinite basis functions, $\Psi(x, y)$,

$$
\phi(x, y)=\sum_{i=1}^{\infty} a_{i} \Psi_{i}(x, y),
$$

where $a_{i}$ is the weighing coefficient of the $i^{\text {th }}$ basis function.

Zernike polynomials, $Z_{i}$, are a set of basis functions commonly used to define optical aberrations and model atmospheric turbulence. ${ }^{24}$ They have the advantage of being $2 \mathrm{D}$ orthonormal and defined on a unit circle. ${ }^{7}$ The Zernike polynomials also represent classical optical aberrations - such as tip, tilt, defocus, astigmatism, etc. at low orders, and are similar to the statistical phase eigenfunctions of Kolmogorov turbulence. ${ }^{24}$

This paper uses the ANSI Standard Z80.28-210 ${ }^{25}$ Zernike polynomial ordering employed by our Thorlabs DM (model: DMP40/M-P01) ${ }^{26}$ and SH-WFS (model: WFS20-5C/M). ${ }^{27}$ Figure 4 shows the first 15 Zernike modes. Piston, $Z_{1}$, only adds a uniform phase shift to the wavefront and thus does not cause image distortion. As a result, it is common to exclude piston from wavefront estimates. The low-order terms $Z_{2}$ and $Z_{3}$, tip and tilt respectively, displace the image at the focal plane. While tip and tilt cause blurring on long-exposure images for a temporal variant wavefront aberration, such as the atmospheric turbulence, they do not affect the spatial resolution of short-exposure images. This paper removes piston, tip, and tilt from wavefront analysis as to focus on the terms which affect the spatial resolution of short-exposure images.

\subsection{Wavefront Reconstruction}

A linear relationship exists between the slope or curvature measurements from the WFS to the coefficients of the Zernike polynomials in Equation (10) by,

$$
\mathbf{m}=\Theta \mathbf{a}+\mathbf{n},
$$

where $\mathbf{m}$ is a vector of the WFS measurements, $\Theta$ is the interaction matrix of the WFS, $\mathbf{a}$ is a vector of the Zernike coefficients, and $\mathbf{n}$ is the additive noise on the measurements. Inverting the equation solves for $\mathbf{a}$. This paper utilizes the least-squared reconstruction algorithm ${ }^{28}$ as defined as,

$$
\hat{\mathbf{a}}=\left(\Theta^{T} \Theta\right)^{-1} \Theta^{T} \mathbf{m},
$$

where $\hat{\mathbf{a}}$ is a vector of the estimated coefficients. 


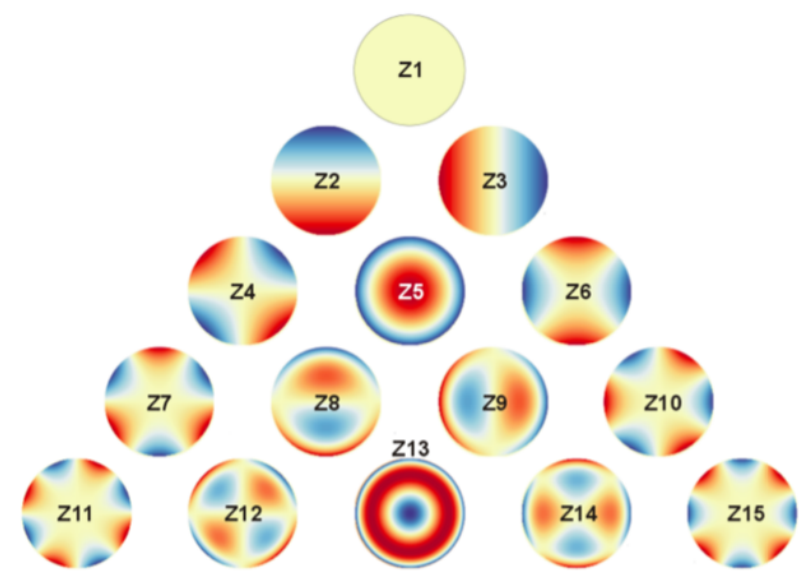

Figure 4: Individual Zernike modes available by our Thorlabs DM. Modes are in ANSI Standard Z80.28-210 ordering. ${ }^{27}$

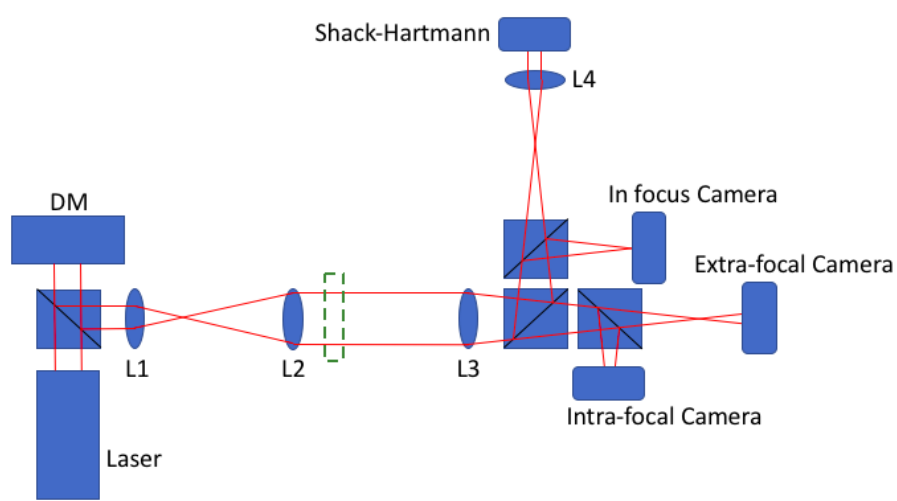

(a) Schematic of the full optical path. A power meter was placed at the dashed green box when measuring the power of the laser.

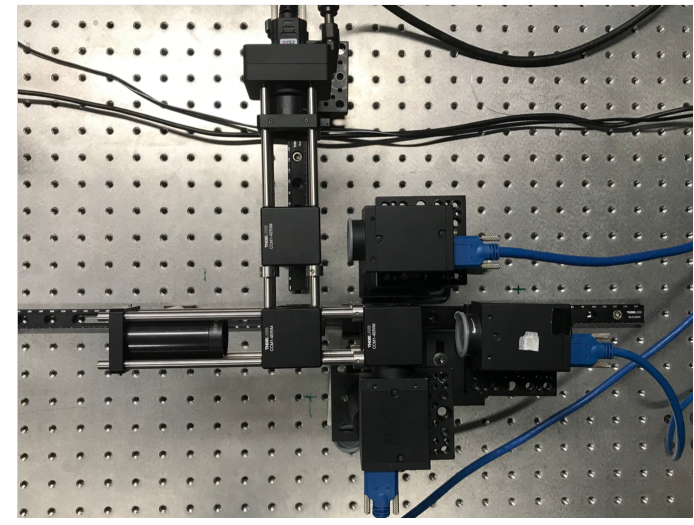

(b) Photo of the test-bench, starting at the refocusing lens, L3.

Figure 5: The three WFS optical test-bench used in the laboratory.

\section{EXPERIMENTAL PROCESS}

Building from the test-bench design by Weddell, ${ }^{29}$ a three WFS optical configuration was assembled in the laboratory, as seen in Figure 5. A collimated laser diode is directed towards a Thorlabs bimorph DM (model: DMP40/M-P01). ${ }^{26}$ The reflected beam is then expanded to twice the original beam diameter using two lenses, and finally re-focused using a $150 \mathrm{~mm}$ lens. The re-focusing lens provides the system with an f-ratio of approximately 6 , which is similar to the $6.3 \mathrm{f}$-ratio of the $0.62 \mathrm{~m} \mathrm{B \& C}$ telescope at MJUO.

The converging beam passes through a series of beam-splitters (BS). The first BS sends half of the signal to the GWFS/CWFS WFS channel, and the other half to the SH-WFS and science (in focus) cameras. A second BS is placed immediately after the first BS in the GWFS/CWFS WFS channel. On either output of the second BS, a Flir Grasshopper3 USB 3.0 camera is placed inside the focal plane of the converging beam and another camera is placed at an equal distance, $l$, outside of the focal plane. These two cameras provide the intra- and extra-focal images needed for the CWFS and GWFS. In the SH-WFS and science channel, a third BS is placed immediately after the first BS. Half of the remaining signal is directed to the science camera at the focal plane, and the other half is re-collimated using a $30 \mathrm{~mm}$ lens and directed at a Thorlabs SH-WFS (model: WFS20-5C/M) ${ }^{27}$

The DM is controlled through the Thorlabs DM GUI. A single Zernike mode (from $Z_{4}-Z_{15}$ ) at +0.4 on the GUI slide-bar is applied to the DM to introduce a wavefront aberration to the system. A burst of 10 frames is captured from each detector using a synchronized external trigger. An image burst is also collected when the 
(a)

\section{(d)}

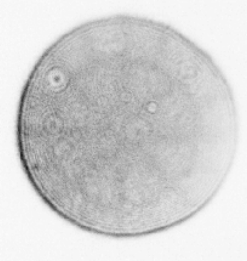

(b)

(c)

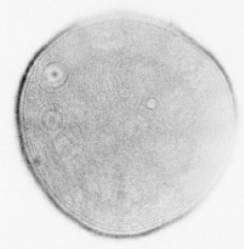

(e)

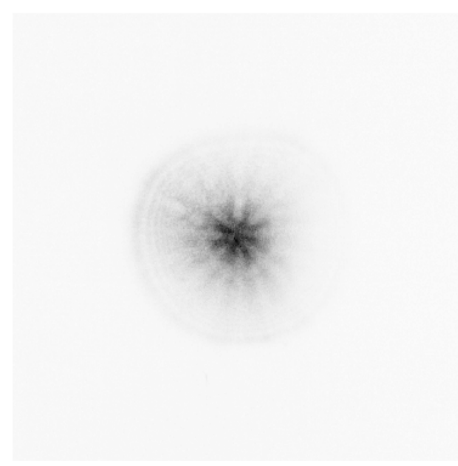

(f)

Figure 6: Sample of images collected from the WFS detectors. Images (a), (b), and (c) were captured with a flat DM wavefront. Images (d), (e), and (f) were captured with a $+0.4 Z_{7}$, trefoil, wavefront applied onto the DM. The left column of images, (a) and (d), are from the SH-WFS, the center column, (b) and (e), are from the intra-focal camera, and the right column, (c) and (f), are from the extra-focal camera.

DM is relaxed, without any wavefront aberrations applied. The data collected while the DM is relaxed, or when the wavefront is theoretically flat, is processed to estimate any internal aberrations of the optical path. The estimated internal aberrations are removed from subsequent WFS estimates of each Zernike mode on the DM using,

$$
\hat{\phi}(x, y)=\hat{\phi}_{\text {aberrated } \mathrm{DM}}(x, y)-\hat{\phi}_{\text {flat } \mathrm{DM}}(x, y)
$$

where $\hat{\phi}_{\text {flat } \mathrm{DM}}(x, y)$ is the estimated wavefront when the DM is relaxed and $\hat{\phi}_{\text {aberrated DM }}(x, y)$ is the estimated wavefront when an aberration is applied to the DM. Figure 6 shows a set of images collected from the WFS cameras with 1) a flat DM and 2) $+0.4 Z_{7}$ (trefoil) on the DM. The individual actuators of the DM can be seen in both defocused images, but are more apparent in the intra-focal image.

The Thorlabs SH-WFS measures the individual centroids of each lenslet and estimates the Zernike coefficients of the wavefront using internal software. The SH-WFS is configured to estimate up to the 15th Zernike coefficient. The average tip and tilt, $Z_{2}$ and $Z_{3}$, is also removed from the wavefront estimate using an internal setting within the Thorlabs GUI. Removing the average tip and tilt eliminates any wedge or misalignment in the optical path which may dominate over the higher order Zernike modes applied to the DM. As a result, the wavefront estimate from the SH-WFS more closely resembles the wavefront applied to the DM. The Thorlabs estimated Zernike coefficients are saved along with the lenslet centroid image. Using Equation (10), the saved coefficients reconstruct the Thorlabs SH-WFS estimated wavefront.

As mentioned in Section 2.3, the extra-focal image, $I_{2}$, needs to be inverted prior to CWFS or GWFS processing. Due to the second BS, which separates the intra- and extra-focal signals, the extra-focal image is 
already inverted along the horizontal axis by the optical path. However, the extra-focal image still needs to be inverted along the vertical axis, which is done after data collection. Once the extra-focal image is inverted, both defocused images, $I_{1}$ and $I_{2}$, are normalized by the total signal intensity and finally processed through the CWFS and GWFS.

For the CWFS, the difference of the signal intensity profiles is measured by subtracting the normalized intrafocal image from the inverted and normalized extra-focal image. The signal intensity difference is converted into a vector, $\mathbf{m}$, which is then processed through Equation (12). The CWFS estimated coefficients for $Z_{1}$, $Z_{2}$, and $Z_{3}$ are manually set equal to zero to remove piston, tip, and tilt from the estimated wavefront phase. Again, removing the first three Zernike coefficients from the estimated wavefront eliminates dominating wavefront aberrations caused by a misalignment in the optical path, and focuses the estimated wavefront on the higher order Zernike modes applied to the DM. The estimated Zernike coefficients are then used to reconstruct the CWFS estimated wavefront from Equation (10). The estimated wavefront with the flat DM, $\hat{\phi}_{\text {flat DM }}(x, y)$, is removed from the aberrated DM wavefront estimate, $\hat{\phi}_{\text {aberrated DM }}(x, y)$, using Equation (13) to eliminate any high order internal aberrations in the optical path.

The GWFS first performs a number of Radon transforms at various angles for both the normalized intrafocal image from the inverted and normalized extra-focal image. A cumulative sum is then calculated across the pixel positions perpendicular to the Radon axis. The horizontal difference is measured between corresponding cumulative sums from the two defocused signals. The cumulative sum and horizontal difference calculations are performed for all of the Radon transform angles. The resulting horizontal differences for the various Radon angles are then converted into a vector, $\mathbf{m}$, and processed through Equation (12). The first three Zernike coefficients are also manually set equal to zero to remove piston, tip, and tilt. Using Equation (10), the estimated Zernike coefficients reconstruct the GWFS estimated wavefront. Any high order internal aberrations in the optical path are removed from the final GWFS wavefront estimate by using Equation (13).

Equation (12) requires an interaction matrix, $\Theta$, for both the CWFS and GWFS in order to estimate the Zernike coefficients of the wavefront. For this paper, each WFS interaction matrix is created synthetically using responses from simulated wavefronts. A wavefront phase is simulated using individual Zernike polynomial modes. The simulated phase is then converted into a complex field using the generalized pupil function, seen in Equation (3), and then virtually propagated using Fresnel diffraction ${ }^{17}$ to generate simulated defocused images. The simulated defocused images are processed through the CWFS and GWFS. The responses from each WFS are recorded into the corresponding synthetic interaction matrix. The simulated defocused images are noiseless, and the recorded WFS measurements are regarded as "perfect" theoretical responses for each WFS. By using simulated wavefronts to generate the interaction matrices, we can compare the real WFS responses to the theory. A theoretical response is recorded into the synthetic interaction matrix for each Zernike mode used to estimate and reconstruct the DM wavefront.

The wavefront phase applied to the DM, $\phi(x, y)$, and the phase estimate from each WFS, $\hat{\phi}(x, y)$, are used to calculate the Mean-Squared-Error (MSE) using,

$$
\mathrm{MSE}=\frac{1}{N_{p i x}} \sum_{(x, y)}^{N_{p i x}}(\phi(x, y)-\hat{\phi}(x, y))^{2},
$$

where $N_{\text {pix }}$ denotes the pixels within the circular aperture of the wavefront reconstruction. The MSE is used to quantify the wavefront estimate of each Zernike mode, $Z_{4}-Z_{15}$, applied to the DM for each WFS.

\subsection{Properties Investigated}

Due to the relatively new interest in the GWFS for astronomical AO and DWFS among the scientific community ${ }^{11-14}$ and the authors' investment into a new imaging instrument for MJUO, ${ }^{15}$ it is important to establish guidelines for various parameters within the GWFS to optimize its performance. Parameters investigated in this paper are: the number of Zernike modes used to construct the synthetic interaction matrix and to estimate the wavefront, the virtual propagation distance used to create the synthetic interaction matrix, the number of Radon angles used to measure the wavefront, and the sensitivity of the WFS under various signal-to-noise ratios (SNR). 
The first property to be investigated is the GWFS's sensitivity to different SNR levels. The power output of the laser is controlled by adjusting the voltage applied across the photo diode. A power meter measures the strength of the laser's signal prior to data collection. The position of the power meter is indicated by the dashed green box in Figure 5(a). Each individual Zernike mode, $Z_{4}-Z_{15}$, is applied to the DM at +0.4 on the Thorlabs GUI slide-bar, and an image burst is collected. The exposure time for each detector is held constant for every laser power level to generate the different SNRs within the images collected. However, the exposure time varies between different modes applied to the DM and different detector types. For example, the intra- and extra-focal detectors have the same exposure time for all laser power levels when $+0.4 Z_{7}$ is applied to the DM, however they have a different exposure time when $+0.4 Z_{8}$ is applied to the DM and also a different exposure time than the SH-WFS detector when $+0.4 Z_{7}$ is applied to the DM. The exposure time for each combined detector type and Zernike mode was determined to avoid saturation at the highest laser power level, while still providing a detectable wavefront signal at the lowest power level. An average laser power of $0.15,0.25,0.35,0.45 \mu \mathrm{W}$ is used to generate the different SNR levels within the WFS images. The SH-WFS has been thoroughly studied over the years, both through simulations and in laboratory settings. As such, equations already exist which describe the SH-WFS's response to different SNR levels,

$$
\begin{gathered}
\sigma_{\theta}=\frac{\theta_{b}}{\mathrm{SNR}} \\
\sigma_{\phi} \approx \frac{2 \pi}{\mathrm{SNR}},
\end{gathered}
$$

where $\sigma_{\theta}$ is the slope measurement error, $\theta_{b}$ is the effective spot size of the low resolution image formed by the SH-WFS lenslet, ${ }^{30}$ and $\sigma_{\phi}$ is the phase estimate error. ${ }^{21}$ However, no such equations have yet been determined for the GWFS. From both formulas in Equation (15), the SH-WFS wavefront estimate improves as the SNR increases. Therefor we would expect the GWFS, as well as the other WFSs, to perform better at higher laser intensities, when the SNR is higher.

The number of Radon angles used to measure the wavefront is also investigated in this paper. It is expected that increasing in the number of Radon angles used to measure the wavefront would improve the wavefront estimate. However, a limit to the achievable improvement would be expected as well, due to an eventual amplification of noise caused by an over-fitting of wavefront measurements. Increasing the number of Radon angles used to measure the wavefront also greatly increases the processing power and time needed to generate an estimate of the phase. This increased processing power and time can hinder AO operations, therefore it is important to find an optimal number of Radon angles used to provide an accurate estimate of the wavefront which can still operate at the frequency needed for an AO system. Pal et al. has used new optimized transforms, instead of the Radon transform, to decrease the processing time needed for the GWFS. ${ }^{12}$ This paper aims to understand the minimum number of Radon angles needed to effectively estimate each single Zernike mode wavefront.

Another property investigated in this paper is the virtual propagation distance used to simulate Fresnel diffraction when creating the synthetic interaction matrices of the GWFS and CWFS. The virtual propagation distance, $z$, is intrinsically related to the physical defocus distance, $l$. Chew calculated the virtual propagation distance to be,

$$
z \approx \frac{f^{2}}{l}
$$

where $f$ is the focal length of the focusing lens or telescope. ${ }^{31}$ For on-sky use of the GWFS, the virtual propagation distance should be equal to the main turbulent altitude of the observational site, and the physical defocus distance should be adjusted accordingly with Equation (16) to obtain the most accurate wavefront estimate. However, Chew also theorized that there is an limit to which $l$ may be operable. He discussed the dangers of rays crossing at large physical propagation distances ${ }^{10}$ as mentioned in Section 2.4. When Chew compared the GWFS and CWFS in pure simulations, he found that the sensitivity of both WFSs increased as the virtual propagation distance also increased, until Fresnel diffraction effects took over. ${ }^{31}$ At short virtual propagation distances, the error in wavefront estimates are dominated by noise in defocused images. We wish to verify Chew's claims by changing $z$ when creating our synthetic interaction matrices and reconstructing the estimated wavefronts in the laboratory. 
The final parameter of interest to optimize is the number of Zernike modes used to estimate the wavefront. The number of modes used to estimate the wavefront is equal to the number of modes used to create the synthetic interaction matrix. Each WFS typically has a general guideline for the optimal amount of modes needed for operation. For the SH-WFS, the number of modes used to effectively estimate and reconstruct the phase aberrations caused by atmospheric turbulence is typically related to the number of lenslets used to subdivide the wavefront signal. For the GWFS, Colodro-Conde et al. argued that the number of Zernike modes should be related to the number of Radon angles used to measure the wavefront. ${ }^{14}$ While we agree with the argument provided by Colodro-Conde et al., we further hypothesize that the number of Radon angles needed to estimate specific Zernike modes in a wavefront is related to the radial order of said Zernike mode. We estimate that the high frequency radial symmetry seen in the different Zernike radial orders may not be detectable until the number of Radon angles is comparable to the frequency of the radial symmetry. Also, Van Dam found that using more than 21 modes to estimate the wavefront in simulations of the GWFS resulted in a worse MSE. ${ }^{9} \mathrm{He}$ theorized the higher Zernike mode coefficients contained more noise than signal. This paper aims to improve the MSE past 21 Zernike modes and to establish a general guideline for the number of Radon angles and Zernike modes used to estimate the wavefront.

\section{RESULTS AND DISCUSSION}

Before various GWFS parameters are investigated and discussed, the three WFSs (GWFS, Thorlabs SH-WFS, and CWFS) are visually compared to each phase aberration applied to the DM to ensure reasonable wavefront reconstructions. All three WFS wavefront estimates, as well as the original Zernike mode applied to the DM, are seen in Table 1. The GWFS is processed using 8 Radon angles and assuming $z=10 \mathrm{~km}$. Visually, the GWFS estimates a wavefront phase similar to the one applied to the DM and estimated by the SH-WFS, however discrepancies were noted in the GWFS with modes $Z_{8}, Z_{9}, Z_{13}$, and $Z_{14}$. Furthermore, the GWFS reconstructs modes $Z_{4}, Z_{6}, Z_{7}, Z_{10}$, and $Z_{11}$ better than the CWFS.

Table 1: Each Zernike mode applied to the Thorlabs DM and the corresponding wavefront phase estimate from each WFS. The first image column are the pure Zernike modes applied to the DM, the second image column are the Thorlabs SH-WFS wavefront estimates, the third image column are the GWFS wavefront estimates, and the fourth image column are the CWFS wavefront estimates. The rows are ordered from Zernike modes $Z_{4}-Z_{15}$. This image series is from a laser power of $0.45 \mu \mathrm{W}$.

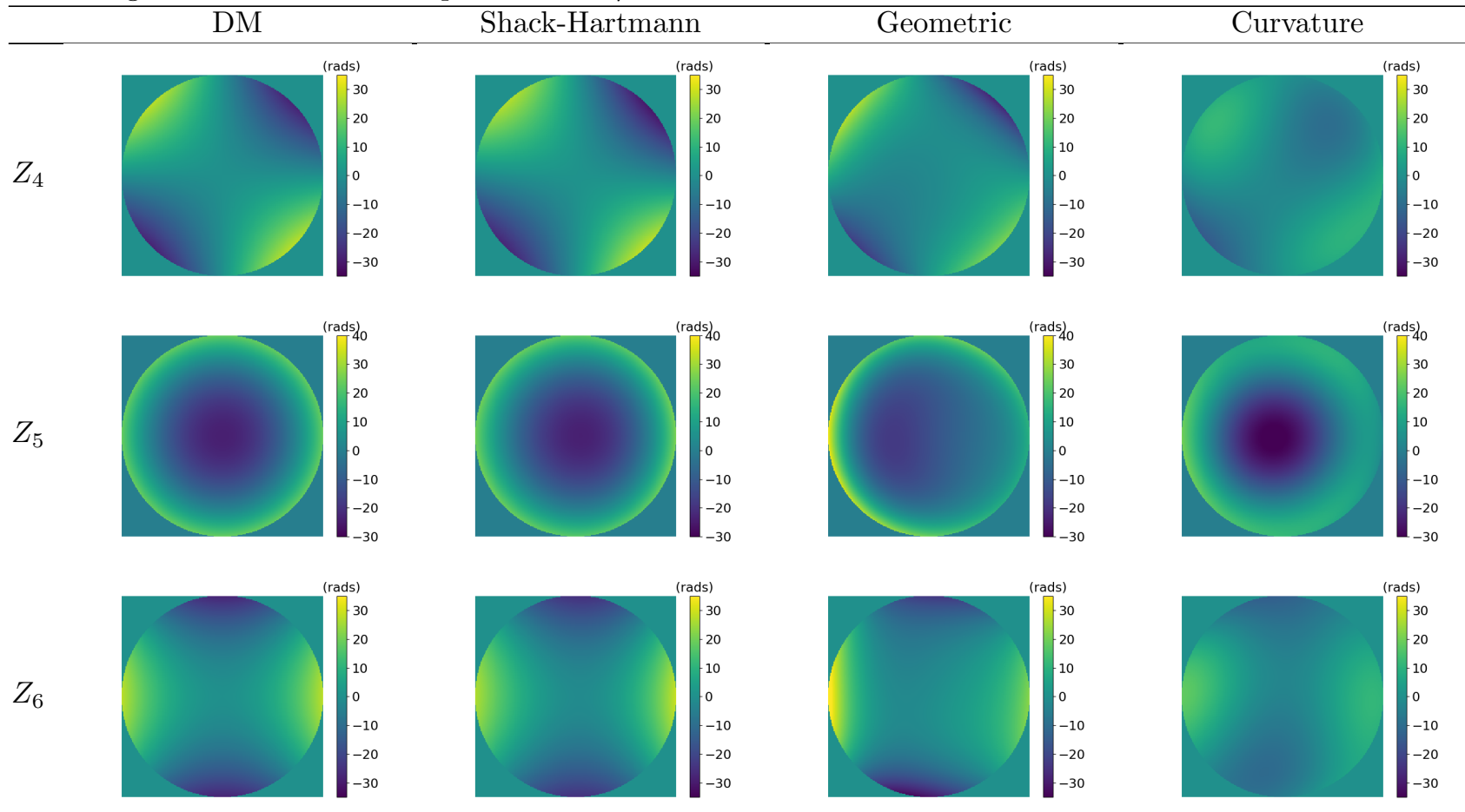


Table 1 - Continued from previous page

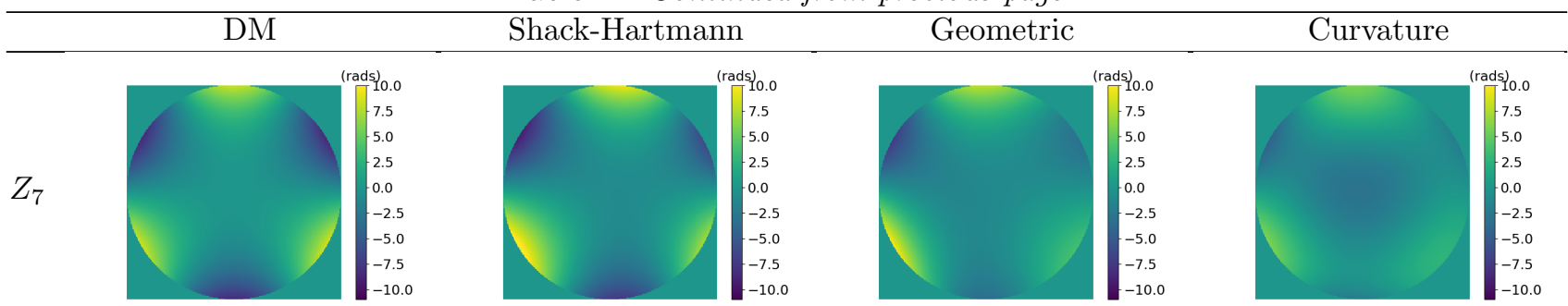
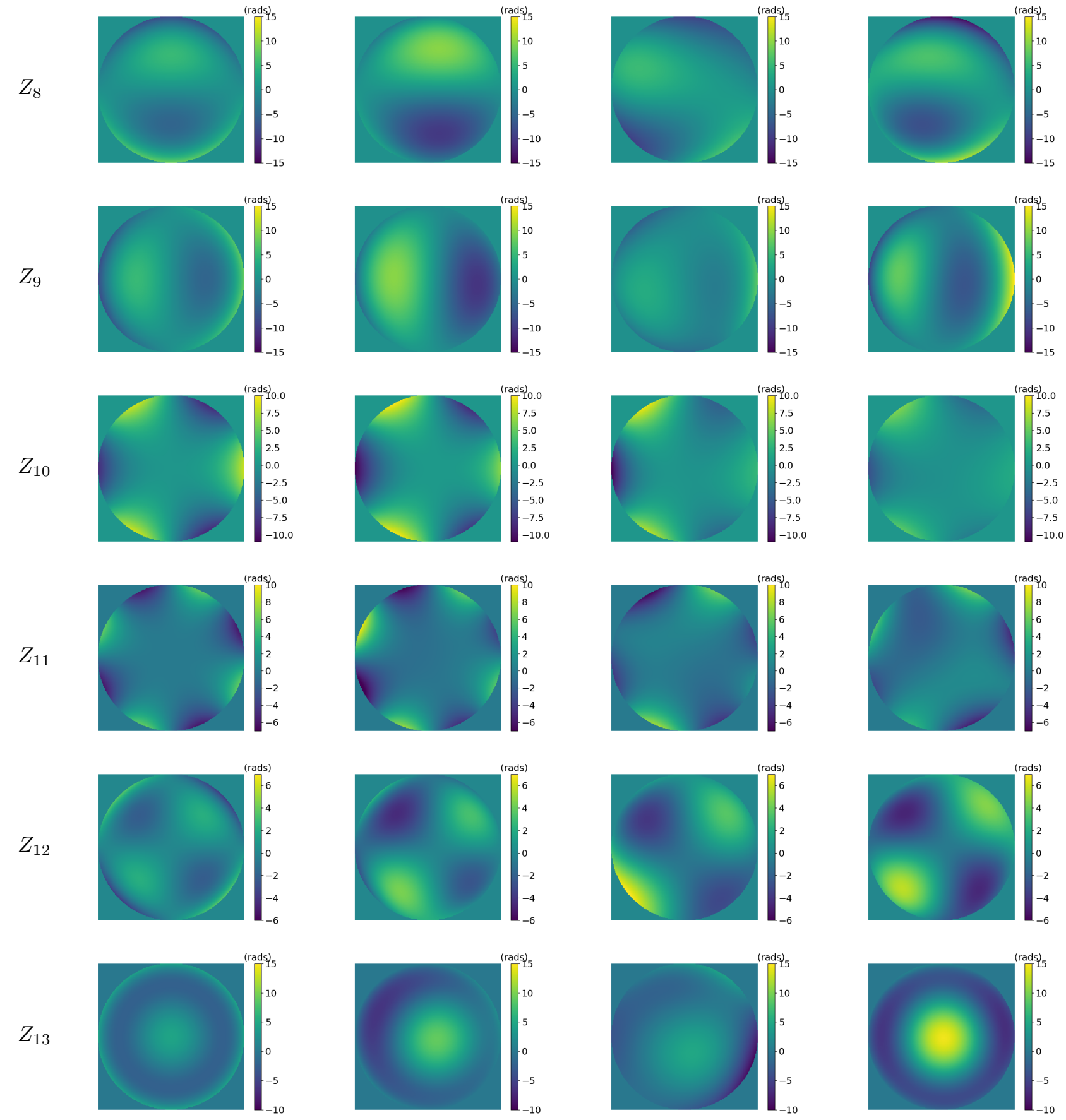
Table 1 - Continued from previous page

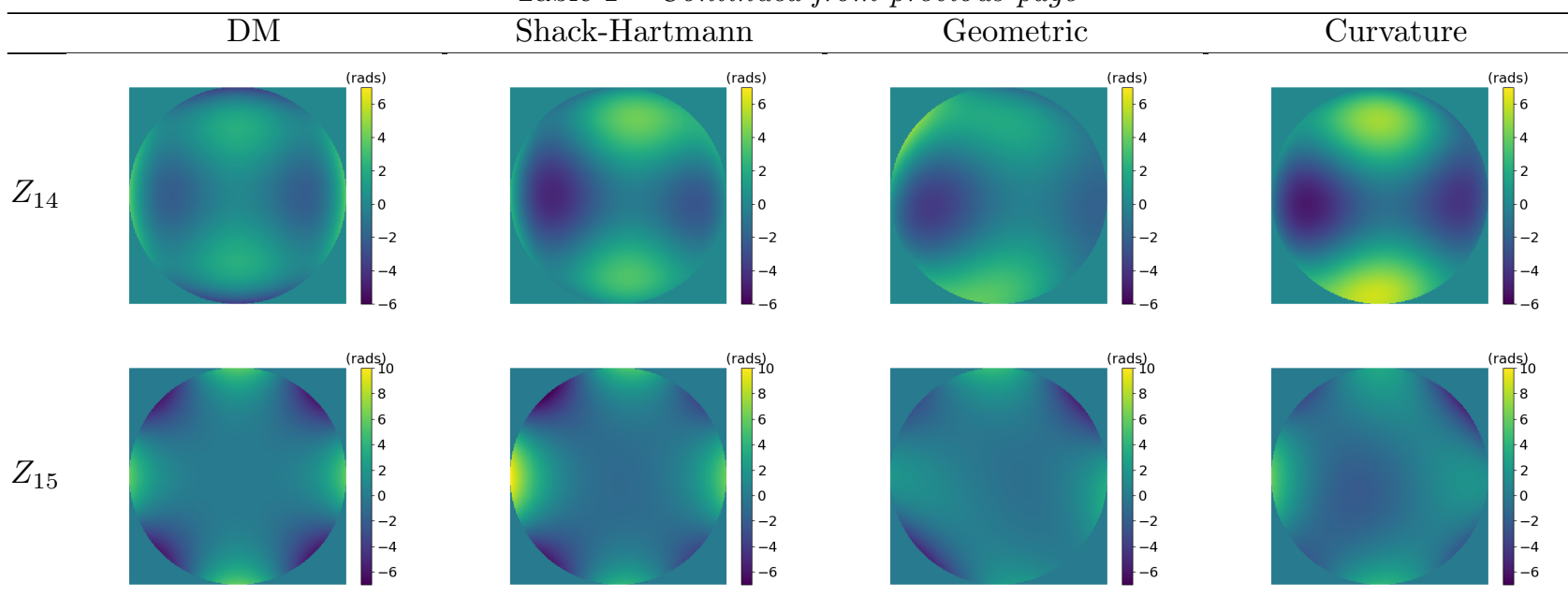

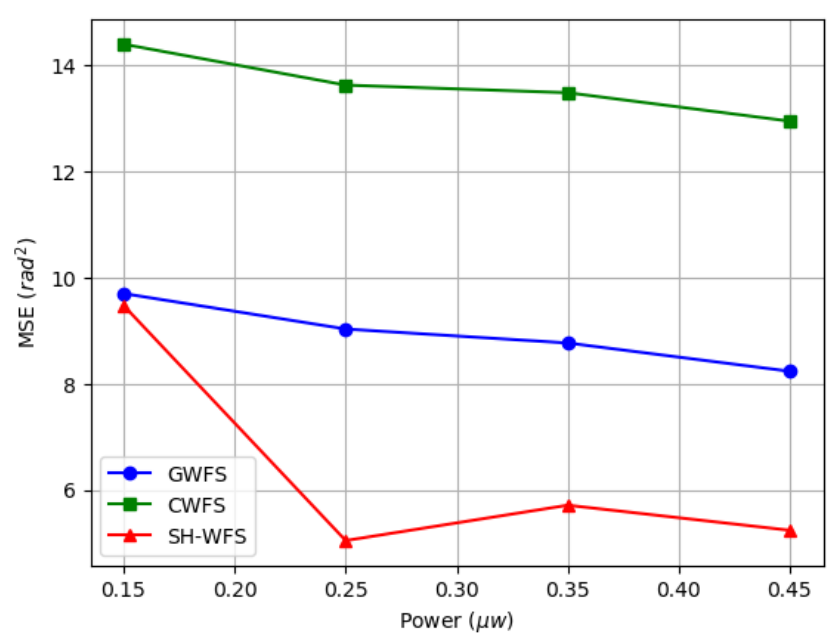

Figure 7: Average MSE $\left(\operatorname{rad}^{2}\right)$ of each WFS for different power levels of the laser.

\subsection{Laser Power}

The MSE is calculated, using Equation (14), for every laser power level, DM shape, and WFS. The virtual propagation distance is assumed to be $10 \mathrm{~km}$ for the GWFS and CWFS, the GWFS used 8 Radon angle measurements, and 15 Zernike modes were used to estimate the wavefronts for all three WFSs. The MSE of each Zernike mode applied to the DM is summed then divided by the total number of modes applied to the DM, $M_{\text {total }}$, to find the average MSE for all three WFSs at each laser power level using,

$$
M S E_{\text {average }}=\frac{1}{M_{\text {total }}} \sum_{\mathbf{p}=4}^{15} M S E_{\mathbf{p}}
$$

where $M S E_{\mathbf{p}}$ is the MSE of mode $\mathbf{p}$ applied to the DM, and $M_{\text {total }}=12$ Zernike modes. The laser power is averaged over 1000 samples from the power meter for each laser setting. The power meter measured $0.15,0.25$, 0.35 , and $0.45 \mu \mathrm{W}$ with an average standard deviation of 0.076 . The resulting average MSE of each WFS at every laser power is shown in Figure 7.

As expected and discussed in Section 3.1, the wavefront estimates improved as the laser intensity increased due to the improved SNR. This is true for both the GWFS and CWFS. However, the SH-WFS experienced the 


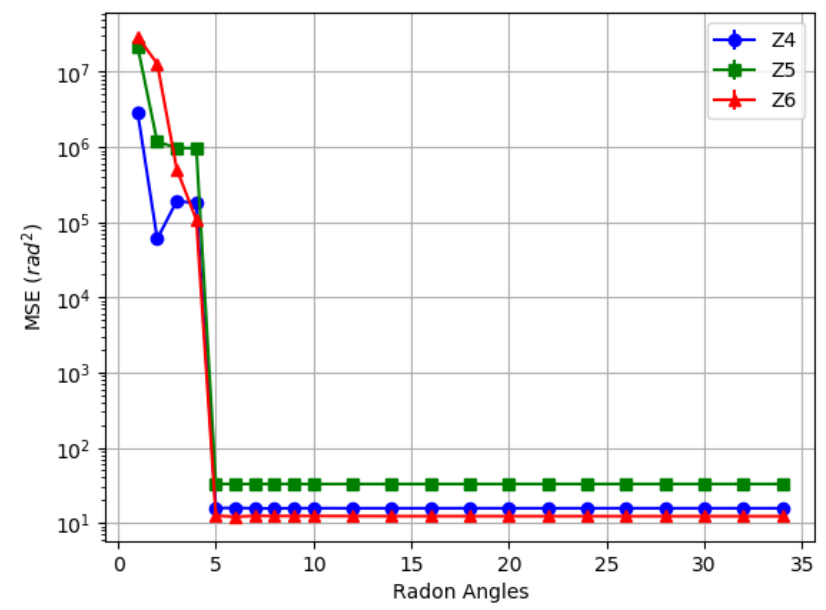

(a)

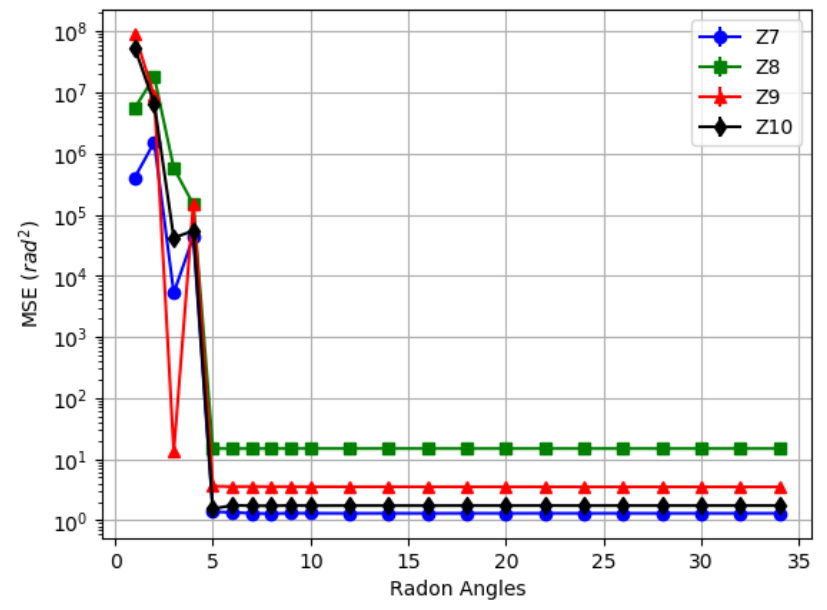

(b)

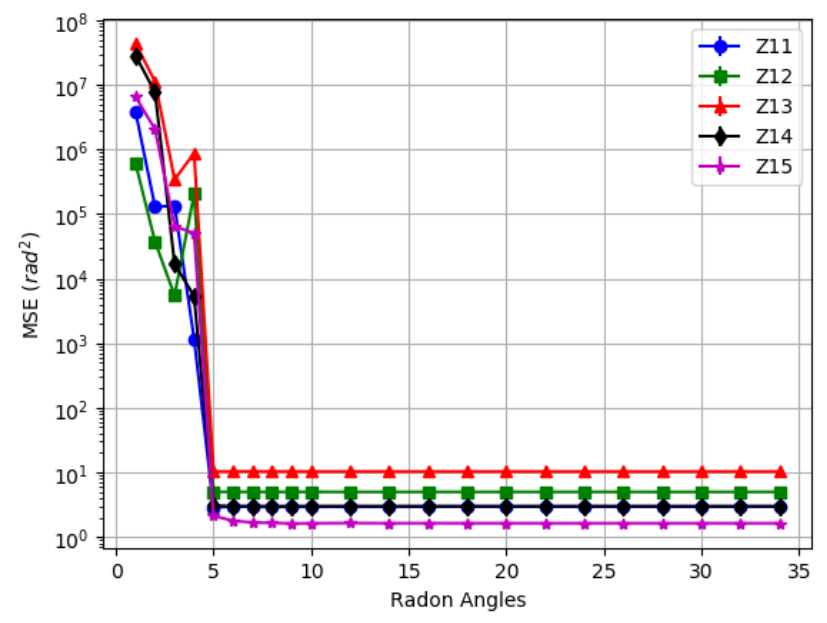

(c)

Figure 8: MSE $\left(\mathrm{rad}^{2}\right)$ of each Zernike mode applied to the DM using a different number of Radon angle measurements in the GWFS wavefront estimate. The graphs are separated by Zernike radial orders, where (a) are the results for the second Zernike radial order, (b) are the results for the third Zernike radial order, and (c) are the results for the fourth Zernike radial order.

best wavefront estimates at $0.25 \mu \mathrm{W}$ instead of $0.45 \mu \mathrm{W}$. Excluding the $0.25 \mu \mathrm{W}$ data point from the SH-WFS, the rest of the SH-WFS measurements agree with the expected trend.

\subsection{Radon Angle}

The defocused images from the $0.35 \mu \mathrm{W}$ power laser data bursts are processed through the GWFS software using a different number of Radon angle measurements. 1-10 Radon angles, incremented by 1, and 12-34 Radon angles, incremented by 2 , are attempted to estimate the wavefronts applied to the DM. The virtual propagation distance is assumed to be $10 \mathrm{~km}$, and 15 Zernike modes were used to estimate the wavefront. The MSE is calculated, again using Equation (14), for the different number of Radon angle measurements. The results of the GWFS MSE for each Zernike mode applied to the DM using a different number of Radon angles are seen in Figure 8. The MSE of the GWFS wavefront estimates drastically improves at 5 Radon angles, and remains stable with more Radon angles. Surprisingly, there does not appear to be a significant improvement to the MSE of the wavefront estimates past 5 Radon angles, nor a deterioration in wavefront estimate quality. 


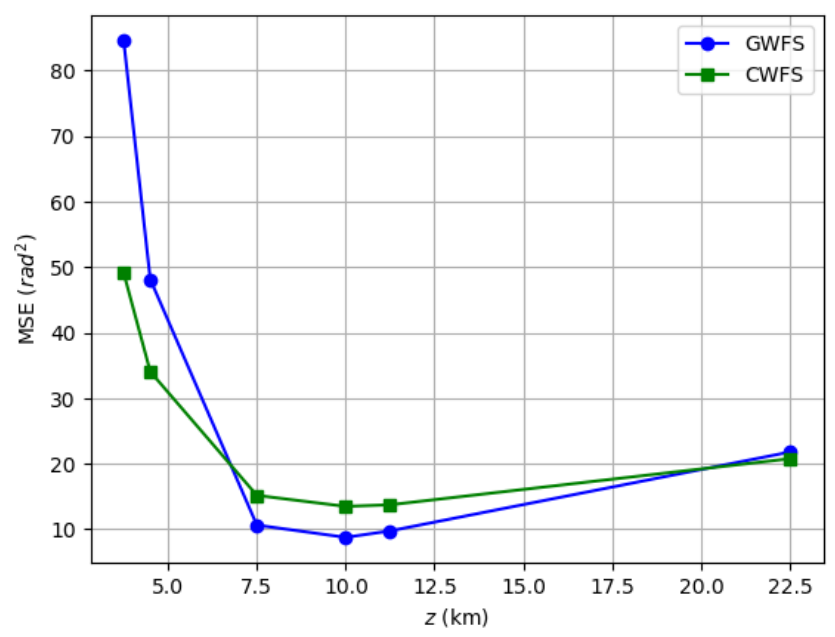

Figure 9: Average MSE ( $\mathrm{rad}^{2}$ ) of the CWFS and GWFS using different virtual propagation distances when creating the synthetic interaction matrices.

The minimum number of angles needed to reconstruct the wavefront phase was anticipated to be related to the radial order of the Zernike mode applied to the DM. However, the expected trend is not seen in the results of this experiment. Of the three Zernike radial orders applied to the DM, all of the wavefront estimates improved and stabilized at the same 5 Radon angles measurement. As discussed in Section 3.1, we also expected the MSE of the wavefront estimate to increase after an excessive number of Radon angles due to an over estimation of detector read noise. However, no such trend was observed.

\subsection{Virtual Propagation Distance}

The virtual propagation distance for the GWFS and CWFS is varied when creating the synthetic interaction matrices. Using the same data series from Section $4.2, z$ is set equal to $3.75,4.5,7.5,10,11.25$, and $22.5 \mathrm{~km}$. The GWFS used 8 Radon angle measurements, and 15 Zernike modes were used to estimate the wavefronts for both the GWFS and CWFS. The MSE is calculated, using Equation (14), for the different virtual propagation distances; then the average MSE is found by using Equation (17). The GWFS and CWFS average MSE results for the different virtual propagation distances are seen in Figure 9.

The results show that the GWFS and CWFSs respond similarly to different propagation distances, and our results agree nicely with Chew. ${ }^{31}$ Both sensors perform poorly at short virtual propagation distances, which according to Equation (16) correspond to large physical defocus distances. At large physical defocus distances, the wavefront is dominated by rays crossing (an issue raised by $\mathrm{Chew}^{10}$ and discussed in Section 3.1). At virtual propagation distances larger than $10 \mathrm{~km}$, the MSE begins to deteriorate again. This is most likely due to geometric optics giving way to diffraction effects caused by Fourier optics at short physical defocus distances.

\subsection{Zernike Modes in Reconstruction}

Finally, the optimal number of Zernike modes used to create the synthetic interaction matrix for each WFS, and subsequently used to estimate and reconstruct each wavefront, is investigated. The number of modes used to estimate the wavefront is incremented by 5 , starting from 10 through 30 modes, using the same data series from Section 4.2. The GWFS used 8 Radon angles, and both the CWFS and GWFS assumed the virtual propagation distance of 10km. The MSE is calculated, using Equation (14), for the different number of Zernike modes; then the average MSE is again found by using Equation (17). The GWFS MSE results of each Zernike mode applied to the DM are seen in Figure 10, and the average MSE results from all three WFSs are shown in Figure 11. The horizontal black line across the top of the Figure 11 indicates the average open loop error of the optical system, which is calculated by

$$
M S E_{\text {open loop }}=\frac{1}{M_{\text {total }}} \sum_{\mathbf{p}=4}^{15} \frac{1}{N_{p i x}} \sum_{(x, y)}^{N_{p i x}} \phi_{\mathbf{p}}(x, y)^{2} .
$$




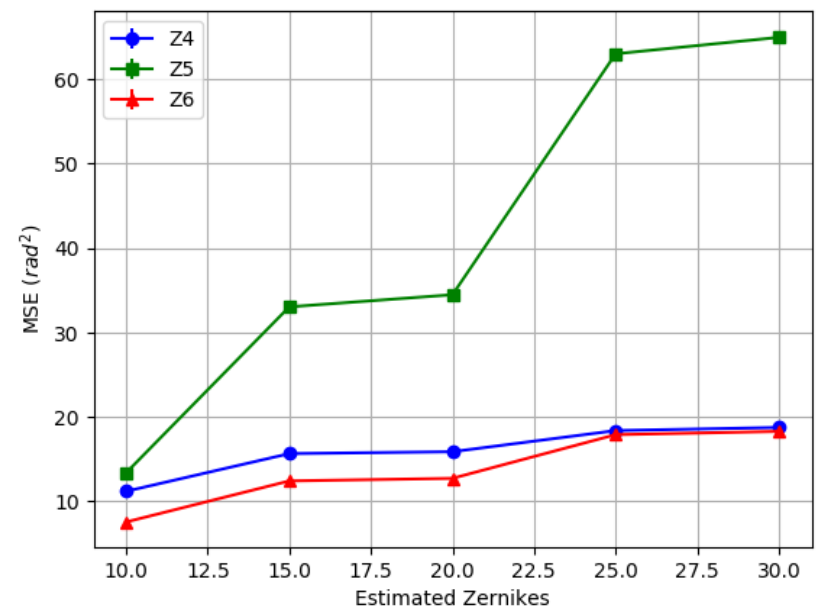

(a)

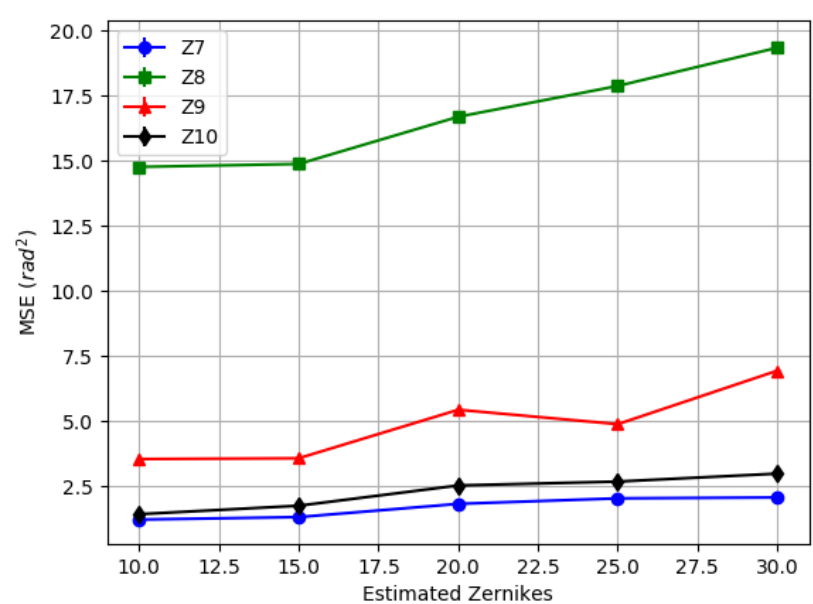

(b)

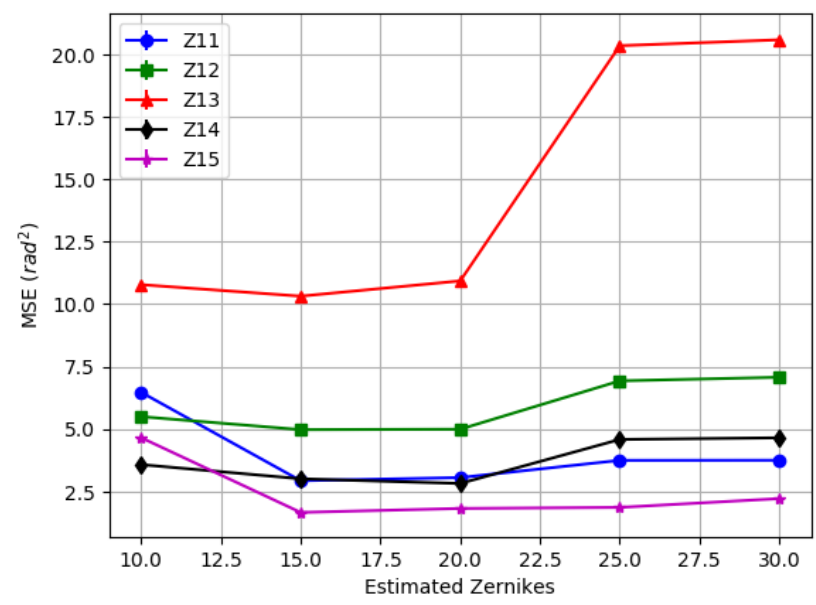

(c)

Figure 10: MSE $\left(\mathrm{rad}^{2}\right)$ from the GWFS of each Zernike mode applied to the DM using a different number of Zernike modes to estimate the wavefront. The graphs are separated by Zernike radial orders, where (a) are the results for the second Zernike radial order, (b) are the results for the third Zernike radial order, and (c) are the results for the fourth Zernike radial order.

The average open loop error is effectively the average MSE of the wavefront without a WFS wavefront estimate correction.

We expected to see a minimum in the MSE of the GWFS at around 20 Zernike modes, as discussed in Section 3.1. While the MSE did worsen past 20 modes, a minimum in the GWFS estimates was not observed. The GWFS actually performed best at 10 modes, and gradually deteriorated as the number of modes increased. The GWFS experiences noise amplification and over-fitting at larger Zernike mode reconstructions.

\section{CONCLUSION}

This paper illustrated and confirmed the GWFS's abilities to estimate and reconstruct single Zernike mode wavefronts. We investigated multiple properties of the GWFS and compared its performance against the CWFS and SH-WFS. Properties investigated included: the sensitivity of the WFSs to different laser power levels and SNRs, an optimal number of Radon angles used by the GWFS, the virtual propagation distance used to the the GWFS and CWFS synthetic interaction matrices, and the number of Zernike modes used by all three WFSs 


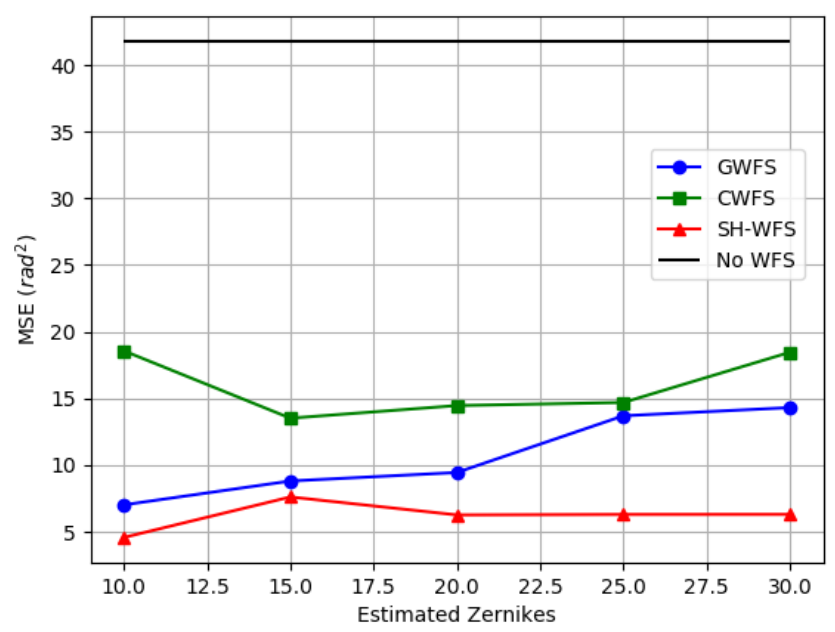

Figure 11: Average MSE $\left(\mathrm{rad}^{2}\right)$ from the GWFS, CWFS, and SH-WFS using a different number of Zernike modes to estimate the wavefront. The horizontal black line across the top of the graph denotes the open loop error of the system.

to estimate and reconstruct the wavefront. As expected, we were able to successfully demonstrate an inverse relationship between the GWFS (as well as the CWFS and SH-WFS) wavefront error to the SNR. We found that the GWFS required at least 5 Radon angles to accurately estimate all of the wavefronts, $Z_{4}-Z_{15}$, applied to the DM. Our hypothesis was that the number of Radon angles needed to reconstruct the wavefront was related to the radial degree of the mode applied to the DM, however this trend was not apparent in our results. When creating the synthetic interaction matrices of the GWFS and CWFS using different virtual propagation distances, our results confirmed previous studies of the GWFS and CWFS. At short virtual propagation distances, the defocused images were dominated by ray crossing; and at long virtual propagation distance, Fresnel diffraction began to take effect. Finally, the GWFS was found to decrease in performance as the number of Zernike modes used to estimate the wavefront increased. This final result was surprising as an increase in reconstruction modes would normally yield to a better estimate of high frequency aberrations, and thus an overall better wavefront estimate. However, the GWFS instead experienced an amplification of read noise as the number of reconstruction modes increased. Overall, the GWFS was able to achieve better wavefront estimations than the CWFS, but was unable to outperform the SH-WFS.

Future works for the GWFS include a further investigation into the number of Zernike modes used to estimate and reconstruct the wavefront (both in simulation and in the laboratory), and the feasibility of atmospheric tomography to reconstruct high definition images of satellites. We aim to increase the optimal number of reconstruction modes used in the GWFS so as to achieve reasonable wavefront estimates of complex wavefronts, such as wavefronts aberrated by the atmospheric turbulence. Currently, there have not been any tests, either simulated or physical, to conclude the viability of atmospheric tomography using the GWFS for the proposed instrument at MJUO in Lake Tekapo, New Zealand. Instead, assumptions have been made based off of the similarities between the GWFS and CWFS. A direct investigation will make a more conclusive argument.

\section{ACKNOWLEDGMENTS}

The authors would like to acknowledge and thank the staff at Mt. John University Observatory. The observational trip to Mt. John was supported by the Marsden Fund Council from Government funding, administered by the Royal Society of New Zealand.

\section{REFERENCES}

[1] Davies, R. and Kasper, M., "Adaptive optics for astronomy," Annual Review of Astronomy and Astrophysics 50, 305-351 (2012). 
[2] Roddier, F., "The effects of atmospheric turbulence in optical astronomy," in [Progress in Optics], Wolf, E., ed., 19, 281-376, Elsevier (1981).

[3] Hickson, P., "Atmospheric and adaptive optics," The Astronomy and Astrophysics Review 22(1), 76 (2014).

[4] Tyson, R. K., [Introduction to adaptive optics], SPIE press (2000).

[5] Primot, J., Rousset, G., and Fontanella, J. C., "Deconvolution from wave-front sensing: a new technique for compensating turbulence-degraded images," JOSA A 7(9), 1598-1608 (1990).

[6] Geary, J. M. et al., [Introduction to wavefront sensors], vol. 18, Spie Press (1995).

[7] Noll, R. J., "Zernike polynomials and atmospheric turbulence," JOSA 66(3), 207-211 (1976).

[8] Roddier, F., "Curvature sensing and compensation: a new concept in adaptive optics," Applied Optics 27(7), 1223-1225 (1988).

[9] van Dam, M. A. and Lane, R. G., "Wave-front sensing from defocused images by use of wave-front slopes," Applied optics 41(26), 5497-5502 (2002).

[10] Chew, T. Y. and Lane, R. G., "Estimating phase aberrations from intensity data," Image and Vision Computing NZ 2003 (2003).

[11] Pal, S., Lambert, A., and Weddell, S., "Comparison of restoration methods for deconvolution from wavefront sensing (DWFS)," in [Adaptive Optics: Analysis, Methods 63 Systems], AOT2C-3, Optical Society of America (2016).

[12] Pal, S., Clare, R., Lambert, A., and Weddell, S., "Slope-based wavefront sensor optimisation with multiresolution analysis," in [Adaptive Optics Systems VI], 10703, 107032Z, International Society for Optics and Photonics (2018).

[13] Hickman, S., Weddell, S., and Clare, R., "Image correction with curvature and geometric wavefront sensors in simulation and on-sky," in [International Conference on Image and Vision Computing New Zealand (IVCNZ)], 1-6, IEEE (2019).

[14] Colodro-Conde, C., Velasco, S., Fernández-Valdivia, J., López, R., Oscoz, A., Rebolo, R., Femenia, B., King, D., Labadie, L., Mackay, C., et al., "Laboratory and telescope demonstration of the tp3-wfs for the adaptive optics segment of AOLI," Monthly Notices of the Royal Astronomical Society 467(3), 2855-2868 (2017).

[15] Weddell, S., Clare, R., and Lambert, A., "Near earth object image restoration with multi-object adaptive optics," in [Proc. 1st NEO and Debris Detection Conference], 22-24 (2019).

[16] Lane, R. G., Tallon, M., Thiebaut, E., and Clare, R. M., "Diffraction-limited image restoration by postcompensation from simultaneous speckle and wavefront sensing observations," in [Adaptive Optical System Technologies II], Wizinowich, P. L. and Bonaccini, D., eds., SPIE Proceedings, 1142, SPIE (2002).

[17] Goodman, J. W., [Introduction to Fourier optics], Roberts and Company Publishers (2005).

[18] Richardson, W. H., "Bayesian-based iterative method of image restoration," JOSA 62(1), 55-59 (1972).

[19] Biggs, D. S. and Andrews, M., "Acceleration of iterative image restoration algorithms," Applied optics 36(8), 1766-1775 (1997).

[20] Van Dam, M. A., Wave-front sensing for adaptive optics in astronomy, PhD thesis, University of Canterbury. Electrical and Computer Engineering (2002).

[21] Clare, R. M., Wavefront sensing and phase retrieval for astronomical imaging, $\mathrm{PhD}$ thesis, University of Canterbury. Electrical and Computer Engineering (2004).

[22] Roddier, F., "Wavefront sensing and the irradiance transport equation," Applied optics 29(10), 1402-1403 (1990).

[23] Liang, Z.-P. and Lauterbur, P. C., [Principles of magnetic resonance imaging: a signal processing perspective], SPIE Optical Engineering Press (2000).

[24] Fried, D. L., "Statistics of a geometric representation of wavefront distortion," JOSA 55(11), 1427-1435 (1965).

[25] Ophthalmics, A. Z.-., "Methods of reporting optical aberrations of eyes: ANSI estandards."

[26] "Piezoelectric deformable mirror dmp40 operation manual," (2020). [Online; posted 21-Mar-2020].

[27] "Optical wavefront sensors (shack-hartmann) operation manual," (2020). [Online; posted 10-Sep-2020].

[28] Roggemann, M. C. and Welsh, B. M., [Imaging through turbulence], CRC press (1996). 
[29] Weddell, S. J. and Lambert, A. J., "Optical test-benches for multiple source wavefront propagation and spatiotemporal point-spread function emulation," Applied optics 53(35), 8205-8215 (2014).

[30] Ellerbroek, B. L. and Tyler, D., "Adaptive optics sky coverage calculations for the gemini-north telescope," Publications of the Astronomical Society of the Pacific 110(744), 165 (1998).

[31] Chew, T. Y., Wavefront sensors in adaptive optics, PhD thesis, University of Canterbury. Electrical and Computer Engineering (2008). 